\title{
Phenanthrene Bioavailability and Toxicity to Daphnia magna in the Presence of Carbon Nanotubes with Different Physicochemical Properties
}

\author{
Florian Zindler, ${ }^{\dagger, \perp}$ Berit Glomstad, ${ }^{\dagger}$ Dag Altin, ${ }^{\ddagger}$ Jingfu Liu, ${ }^{\S}$ Bjørn M. Jenssen, ${ }^{\dagger}$ and Andy M. Booth ${ }^{*}, \|$ \\ ${ }^{\dagger}$ Department of Biology, Norwegian University of Science and Technology, Trondheim NO-7491, Norway \\ "BioTrix, Trondheim NO-7022, Norway \\ ${ }^{\S}$ State Key Laboratory of Environmental Chemistry and Ecotoxicology, Research Center for Eco-Environmental Sciences, Chinese \\ Academy of Sciences, Beijing 100085, China \\ "SINTEF Materials and Chemistry, Trondheim NO-7465, Norway
}

Supporting Information

\begin{abstract}
Studies investigating the effect of carbon nanotubes (CNTs) on the bioavailability and toxicity of hydrophobic organic compounds in aquatic environments have generated contradictory results, and the influence of different CNT properties remains unknown. Here, the adsorption of the polycyclic aromatic hydrocarbon phenanthrene $(70-735 \mu \mathrm{g} / \mathrm{L})$ to five types of CNTs exhibiting different physical and chemical properties was studied. The CNTs were dispersed in the presence of natural organic matter (nominally $20 \mathrm{mg} / \mathrm{L}$ ) in order to increase the environmental relevance of the study. Furthermore, the bioavailability and toxicity of phenanthrene to Daphnia magna in the absence and presence of dispersed CNTs was investigated. Both CNT dispersion and adsorption of phenanthrene appeared to be influenced by CNT physical properties (diameter and specific surface area). However,

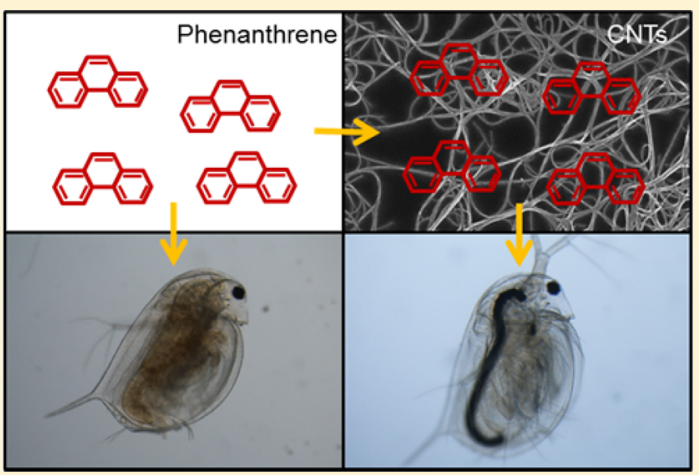
dispersion and phenanthrene adsorption was not influenced by CNT surface chemical properties (surface oxygen content), under the conditions tested. Based on nominal phenanthrene concentrations, a reduction in toxicity to D. magna was observed during coexposure to phenanthrene and two types of CNTs, while for the others, no influence on phenanthrene toxicity was observed. Based on freely dissolved concentrations, however, an increased toxicity was observed in the presence of all CNTs, indicating bioavailability of CNT-adsorbed phenanthrene to D. magna.
\end{abstract}

\section{INTRODUCTION}

The production of carbon nanotubes (CNTs) is increasing due to their incorporation into a growing number of applications, including in composite materials and environmental technologies. ${ }^{1,2}$ Due to their hydrophobicity and tendency to aggregate and settle, a significant proportion of CNTs released into the environment are assumed to accumulate in sediments. ${ }^{3}$ However, the adsorption of colloidal and dissolved natural organic matter (NOM) to CNT surfaces has been found to increase the dispersion stability and water column residence time of CNTs in aqueous media, significantly influencing their environmental fate and behavior. ${ }^{4-8}$ CNTs are potent adsorbents of hydrophobic organic compounds (HOCs), making them interesting e.g. in remediation of contaminated sites or in wastewater treatment. ${ }^{2,9,10}$ This raises questions with respect to their effect on the fate, bioavailability, and toxicity of HOCs, including polycyclic aromatic hydrocarbons (PAHs) which are common pollutants in natural environments. CNT adsorption of HOCs is influenced by several factors, including the CNT physicochemical properties, CNT dispersion state, the presence of NOM, and media properties. ${ }^{11-15}$
Several studies have observed ingestion of CNTs by aquatic organisms, including daphnids and fish. ${ }^{16-19}$ Moreover, adverse effects as a direct result of CNT exposure have been observed in algae, daphnids, and fish. ${ }^{4,17,18,20-23}$ Further studies have investigated the adsorption of HOCs by CNTs or other carbon-based nanomaterials (CNMs), such as fullerene $\mathrm{C}_{60}$, and the subsequent bioavailability and toxicity of these contaminants to aquatic organisms. Only freely dissolved HOCs are considered bioavailable to aquatic organisms, and the adsorption to CNMs has been suggested to reduce HOC bioavailability. However, studies have shown contradictory findings. Some studies found that CNM adsorption of HOCs led to a reduction in their bioavailability and toxicity to aquatic organisms. $^{24-26}$ In contrast, other studies have reported that HOCs adsorbed by CNMs remain bioavailable to both pelagic and benthic organisms. ${ }^{11,19,25,27,28}$ For example, in Japanese

Received: June 29, 2016

Revised: August 25, 2016

Accepted: October 4, 2016

Published: October 4, 2016 
Medaka (Oryzias latipes) it was demonstrated that a CNTadsorbed PAH (phenanthrene) was released after ingestion of the particles, resulting in increased body concentrations in the fish. ${ }^{19}$ Our previous study revealed that a range of CNTs with different physicochemical properties readily adsorbed phenanthrene in the presence of environmentally relevant concentrations of NOM and that it was bioavailable to freshwater algae (Pseudokirchneriella subcapitata). ${ }^{11}$ These results show that CNMs can facilitate the transport of coadsorbed contaminants into organisms through alternative uptake routes, indicating that they remain bioavailable. The bioavailability of CNTadsorbed HOCs is thought to be influenced by factors such as CNT aggregation behavior and the presence of NOM. Aggregation behavior is believed to affect CNT retention/ elimination rates in organisms, while the presence of NOM has been suggested to impede PAH desorption from CNTs. ${ }^{19,24}$ The contradictory results from previous studies highlight the complexity of combined studies with CNTs and HOCs, and how the results may be influenced by CNT properties or environmental factors (e.g., NOM). Despite the fact that CNT properties can influence CNT aggregation behavior ${ }^{6}$ and their adsorption of organic pollutants, ${ }^{1-13}$ few studies have addressed the effect of CNT properties on cocontaminant bioavailability and toxicity to aquatic organisms. More knowledge regarding CNT effects on bioavailability and toxicity of HOCs in the presence of NOM is of importance for the evaluation of their environmental effects.

In this study, we used phenanthrene as a model PAH to evaluate the bioavailability and toxicity to $D$. magna in the presence of CNTs and environmentally relevant concentrations of NOM. To investigate the influence of the physicochemical properties of CNTs on the bioavailability and toxicity of phenanthrene, five types of CNTs were used in the study, including one single-walled CNT (SWCNTs), two nonfunctionalized multiwalled CNTs (MWCNT-15 and MWCNT-30) with different diameter and lengths and two functionalized MWCNTs (hydroxyl: MWCNT-OH and carboxyl: MWCNT-COOH). Adsorption of phenanthrene to the different types of CNTs in the presence of NOM was determined, and the subsequent influence on bioavailability and toxicity to $D$. magna assessed through acute toxicity tests.

\section{MATERIALS AND METHODS}

Chemicals. The carbon nanotubes (SWCNT, MWCNT-15, MWCNT-30, MWCNT-OH, and MWCNT-COOH; > 95\%) were obtained from Timesnano (Chengdu Organic Chemicals Co., Ltd., Chinese Academy of Sciences). Characterization of the CNTs was performed to determine outer diameter, purity, specific surface area (SSA), and surface oxygen content. Details on the characterization techniques have been previously published. ${ }^{11}$ Phenanthrene ( $\geq 99.5 \%$, Sigma-Aldrich) was dissolved in methanol (analytical grade, Fluka Analytical) for the preparation of stock solutions $(100 \mathrm{mg} / \mathrm{L})$. Suwannee River natural organic matter (SR-NOM) was purchased from the International Humic Substance Society (IHSS).

Preparation of CNT Dispersions. Elendt M7 medium ${ }^{29}$ containing $20 \mathrm{mg} / \mathrm{L}$ SR-NOM (M7-NOM) was used as medium for the preparation of CNT dispersions throughout the study. SR-NOM was dissolved in M7 medium by magnetic stirring for $24 \mathrm{~h}$. Undissolved material was subsequently removed by filtration through a $0.2 \mu \mathrm{m}$ Nalgene filter unit (Thermo Fisher Scientific, Inc.). The $\mathrm{pH}$ of the M7-NOM was $7.74 \pm 0.08$ and needed no adjustment after preparation. CNT stock dispersions in M7-NOM (100 mg/L) were prepared using bath sonication according to a previously published method. ${ }^{11}$ After sonication, the stock dispersions were mixed into larger volumes of the same media to achieve an initial CNT concentration of $10 \mathrm{mg} / \mathrm{L}$. The prepared dispersions were left for $24 \mathrm{~h}$ to allow any nondispersed CNT aggregates to settle out of the water column before the supernatant containing the remaining dispersed CNTs was withdrawn and used in the further experiments. The CNT concentration in the supernatant was determined by UV-vis absorbance measurements at $800 \mathrm{~nm}$ (Hitachi U-2000 Spectrophotometer), as no interference from NOM, the media, or phenanthrene was observed at this wavelength. Quantification was performed using calibration curves prepared for individual CNTs as previously described. ${ }^{11}$ The calibration curves are presented in Figure S1 in the Supporting Information (SI). For MWCNT15, MWCNT-OH, and MWCNT-COOH the concentration was determined in single replicates which were subsequently used throughout the adsorption and toxicity studies. Due to an oversight, the concentration of MWCNT-30 and SWCNT was not measured directly in the dispersions used in the adsorption and toxicity studies. The concentration of SWCNT and MWCNT-30 was therefore determined independently in seven and six replicates, respectively. The average concentrations of these were used to confidently estimate the CNT concentration in dispersions used in adsorption and toxicity studies. The coefficients of variation $(\mathrm{CV})$ of the replicates were $15 \%$ and $27 \%$ for SWCNT and MWCNT-30, respectively.

Phenanthrene Toxicity to Daphnids. D. magna were cultivated in Elendt M7 medium at $20 \pm 2{ }^{\circ} \mathrm{C}$. Prior to testing, they were kept under a 16 -h light and 8 -h dark cycle and fed with the freshwater algae P. subcapitata. A modified version of the OECD Daphnia sp., Acute Immobilisation Test ${ }^{29}$ was used to assess phenanthrene toxicity to daphnids, using 3-4 day old daphnids. Pretests showed that for newborn organisms $(<24 \mathrm{~h}$, as recommended in the OECD test guidelines) attachment of CNT aggregates to the animals could cause mechanical immobilization. Although mechanical immobilization could be considered as a physical mechanism of toxicity, it is suggested this would not be of significant relevance in natural fresh waters where CNT concentrations are expected to be low. To minimize the risk of mechanical influence of the CNTs, larger 3-4 day old animals were used.

The daphnids were exposed to phenanthrene spiked into M7-NOM at five different concentrations $(70 \mu \mathrm{g} / \mathrm{L}, 126 \mu \mathrm{g} / \mathrm{L}$, $227 \mu \mathrm{g} / \mathrm{L}, 408 \mu \mathrm{g} / \mathrm{L}$, and $735 \mu \mathrm{g} / \mathrm{L})$ in order to determine the effect concentrations. Five animals were carefully pipetted into $20 \mathrm{~mL}$ exposure solution contained in $22 \mathrm{~mL}$ glass vials with PTFE lined screw caps (Agilent Technologies, Inc.), maintaining a headspace of $1-2 \mathrm{~cm}$. Four replicates were included at each phenanthrene concentration. The animals were exposed for $48 \mathrm{~h}$ in darkness at a temperature of $19.6 \pm 0.3{ }^{\circ} \mathrm{C}$, after which the number of immobilized animals was counted. Abnormalities, including mortality and disoriented animals, were also recorded. Negative controls for M7 $(n=8)$, M7NOM $(n=32)$, and methanol dissolved in M7-NOM (which was used as a solvent for phenanthrene, $7.35 \mu \mathrm{g} / \mathrm{L} ; \mathrm{n}=8$ ) were included in the study. Dissolved oxygen $\left(\mathrm{dO}_{2}\right)$ and $\mathrm{pH}$ were determined in the sample solutions at the start and end of the exposure.

A total of six tests with phenanthrene-only (i.e., without the presence of CNTs) were performed in order to investigate the variability of the test. Four of the tests were performed using 
the same batch of daphnids, while two tests were performed with different batches of the daphnids, giving very good control of test variability both within one batch and between different batches of daphnids. Based on these data, effect concentrations causing $50 \%$ immobilization of D. magna $\left(\mathrm{EC}_{50}\right)$ resulting from exposure to phenanthrene-only were calculated. Furthermore, the $\mathrm{EC}_{30}$ and $\mathrm{EC}_{70}$ values were calculated and used as a control for biological variability throughout the rest of the study (i.e., in the tests subsequently performed with CNTs). This was done by exposing daphnids to phenanthrene-only at the concentrations corresponding to the calculated $\mathrm{EC}_{30}$ and $\mathrm{EC}_{70}$ values and verifying that the responses were similar in all tests. The calculations and statistical analysis were performed using GraphPad Prism 6.0. EC values were calculated from nonlinear fit/concentration-response stimulation ( $\log$ (agonist) vs response), using an ordinary fit (least-squares) and variable slope, with bottom and top constrain set as $0 \%$ and $100 \%$, respectively. Statistical differences between groups were tested using analysis of variance (ANOVA) with Tukey's post hoc test.

Phenanthrene Toxicity in the Presence of CNTs. The CNT dispersions were prepared as described, spiked with five nominal concentrations $\left(C_{\text {nominal }}\right)$ of phenanthrene $(70-735$ $\mu \mathrm{g} / \mathrm{L}$ ) and divided into aliquots of $20 \mathrm{~mL}$ contained in $22 \mathrm{~mL}$ glass vials. The phenanthrene $C_{\text {nominal }}$ range used was identical to that of the test with pure phenanthrene (no CNTs). The samples were left on an orbital shaker $(165 \mathrm{rpm})$ in darkness for 5 days to ensure adsorption equilibrium. ${ }^{1,30}$ Once equilibrium had been achieved, five daphnids were placed in each sample vial and exposed for $48 \mathrm{~h}$ under identical conditions as those used in the phenanthrene-only toxicity test described above. Four replicates at each $C_{\text {nominal }}$ were used. Immobilization and abnormalities (CNT attachment to the animals, mortality and disoriented animals) were recorded after the $48 \mathrm{~h}$. Controls containing only CNTs (CNT dispersions without phenanthrene) were included to account for any toxicity due to the CNTs themselves. In addition, animals were exposed to phenanthrene-only at the calculated $\mathrm{EC}_{30}$ and $\mathrm{EC}_{70}$ from the phenanthrene-only tests to control for any biological variability (as previously described).

Microscopy Analysis. Visual inspections of the exposed daphnids, in the absence and presence of CNTs, were performed using light microscopy (Leica MZ-APO microscope; Leica Microsystems, with a DS-5M-U1 camera; Nikon).

Chemical Analysis. The concentration of freely dissolved phenanthrene $\left(C_{\text {free }}\right)$ was determined at the start of the exposure for all phenanthrene concentrations, both in the absence and presence of CNTs. Three replicates were analyzed in the absence of CNTs, while single replicates were analyzed in the presence of CNTs. Duplicate measurements of each replicate were applied in all cases. In studies where CNTs were present, filtration using a hydrophilic polytetrafluoroethylene (PTFE) filter membrane $(0.1 \mu \mathrm{m}$ pore size, Merck Millipore) was used to isolate and remove the dispersed CNTs from the water phase prior to determination of $C_{\text {free }}$. Details on the filtration technique have been previously published. ${ }^{11,31}$ Briefly, a highly reproducible sorption of phenanthrene to the filter membrane of approximately $18 \%$ was observed at all phenanthrene concentrations. ${ }^{31}$ This allowed for the calculation of phenanthrene loss from the dissolved phase during the filtration process. $C_{\text {free }}$ in the presence of CNTs was then used to calculate the phenanthrene adsorption to CNTs. $C_{\text {free }}$ was determined by direct injection using high-performance liquid chromatography with photodiode-array UV detection (HPLC-
UV; Agilent Technologies, Inc.) as previously described. ${ }^{31}$ The Dubinin-Ashtakhov model (DAM), which was successfully used for describing phenanthrene adsorption to CNTs in our previous study, was fitted to the adsorption data. ${ }^{11}$

\section{RESULTS AND DISCUSSION}

CNT Properties and Dispersion Behavior. A detailed summary of the CNT physicochemical properties determined have been previously published ${ }^{11}$ and is available in Table S1. Briefly, transmission electron microscopy analysis indicated that the SWCNT had an average diameter of $\sim 2 \mathrm{~nm}$, while the four MWCNTs had average diameters that ranged between 14 and $20 \mathrm{~nm}$. The SWCNT exhibited a SSA of $483.7 \mathrm{~m}^{2} / \mathrm{g}$, significantly higher than the MWCNTs with SSA values $<177.4 \mathrm{~m}^{2} / \mathrm{g}$. Surface oxygen content was $5.7 \%$ and $3.9 \%$ for MWCNT-COOH and MWCNT-OH, respectively, while the nonfunctionalized CNTs all had similar surface oxygen contents of $<2 \%$.

The concentration of the five different CNTs remaining dispersed in the supernatants after the $24 \mathrm{~h}$ settling period was determined, and these values were used to calculate the available surface area in each dispersion (Table 1). The

Table 1. CNT Dispersed Concentration in M7-NOM and Calculated Available Surface Area

\begin{tabular}{lccc} 
& $\begin{array}{c}\text { dispersed concn } \\
(\mathrm{mg} / \mathrm{L})\end{array}$ & $\begin{array}{c}\text { percentage } \\
\text { dispersed }(\%)\end{array}$ & $\begin{array}{c}\text { available surface area } \\
\left(\mathrm{m}^{2} / \mathrm{L}\right)\end{array}$ \\
SWCNT & 0.46 & 4.6 & 0.22 \\
MWCNT-15 & 2.45 & 24.5 & 0.34 \\
MWCNT-30 & 2.62 & 26.2 & 0.46 \\
MWCNT- & 2.29 & 22.6 & 0.32 \\
$\quad$ OH & & & \\
MWCNT- & 2.41 & 24.1 & 0.34 \\
COOH & & & \\
\hline
\end{tabular}

available surface area in each dispersion is calculated based on the individual SSA values determined for the dry CNTs rather than dispersed CNTs and can therefore only be considered as estimates. However, as surface area is likely to be an important factor in controlling adsorption and to have a potentially decisive effect on cocontaminant bioavailability and toxicity, ${ }^{11,13,32}$ these estimates offer a useful measure for interpreting the results generated in the subsequent toxicity studies.

The SWCNT and MWCNTs exhibited large differences in dispersibility, with the percentage of the original $10 \mathrm{mg} / \mathrm{L}$ of each CNT remaining in dispersion being 5\% SWCNT, 25\% MWCNT-15, 26\% MWCNT-30, 23\% MWCNT-OH, and 24\% MWCNT-COOH. The SWCNT exhibited a lower dispersed concentration than the MWCNTs, consistent with the observations made in our previous study ${ }^{11}$ and in accordance with studies reporting a positive relation between CNT diameter and dispersibility. ${ }^{6}$ The dispersion concentrations for the four functionalized and nonfunctionalized MWCNTs were all very similar, being in the range $2.29-2.62 \mathrm{mg} / \mathrm{L}$ (23$26 \%$ ). Interestingly, this is in contrast to our previous study using the same CNTs in algae medium with NOM, where higher dispersed concentrations were observed for the functionalized MWCNTs than for the nonfunctionalized MWCNTs. ${ }^{11}$ Previous studies have shown a positive relationship between surface oxygen content and dispersibility. ${ }^{6}$ However, artificial freshwater containing high concentrations of divalent cations and NOM has been shown to reduce the effect of CNT surface oxygen on dispersibility. ${ }^{33}$ The Elendt 
M7 medium used in the current study is more complex and has a higher ionic strength and concentration of divalent cations than the algae medium used in our previous study. ${ }^{11}$ The specific conductivity (measure of ionic strength) for Elendt M7 and the algae medium is 658 and $161 \mu \mathrm{S} / \mathrm{cm}$, respectively, while the $\mathrm{Ca}^{2+}$ concentration (calculated from the media composition $^{29,34}$ ) is 2.0 and $0.1 \mathrm{mM}$, respectively. As the same CNTs, dispersion method, and NOM concentration were employed in both studies, it is suggested that the Elendt M7 medium composition is causing the comparable dispersion concentrations for functionalized and nonfunctionalized MWCNTs. It is therefore important to consider the role of aquatic ecotoxicity media recommended for different species in standard tests. This also highlights the importance of such parameters in natural waters, and the role they will have on CNT behavior in the aquatic environment.

Phenanthrene Adsorption to CNTs. The determination of phenanthrene $C_{\text {free }}$ showed significant phenanthrene adsorption to all five types of CNTs. When present at their individual maximum dispersion concentrations shown in Table 1, the five CNTs adsorbed $25-50 \%$ of the $C_{\text {nominal }}$ phenanthrene over the concentration range tested (70-735 $\mu \mathrm{g} / \mathrm{L}$; Figure S2). The adsorption isotherms are presented in Figure 1, and adsorption capacity and adsorption affinity for
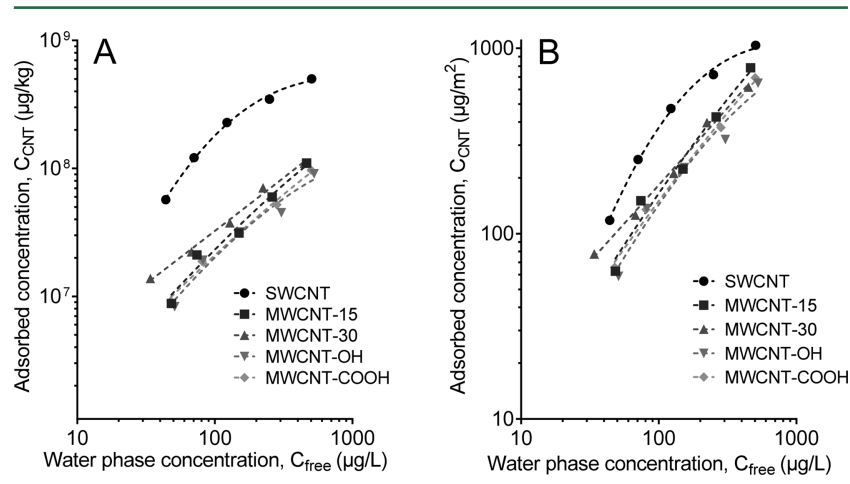

Figure 1. Adsorption isotherms of phenanthrene adsorbed to five types of CNTs calculated based on A) CNT mass and B) CNT available surface area.

each of the CNTs, described by the DAM parameters $\log Q^{0}$ and $E$, respectively, are presented in Table S2. Furthermore, $C_{\text {free }}$ as a function of nominal phenanthrene concentrations $\left(C_{\text {nominal }}\right)$ is presented in Figure $S 3$. As the adsorption data used for model fitting was based on single data points, no statistical tests of the data were performed. The use of single data points was based upon the following: (i) high reproducibility was observed between the replicates from adsorption studies in our previous study employing the same method, ${ }^{11}$ (ii) the DAM provided a strong fit for the single data points generated in the current study, and (iii) the calculated maximum adsorption capacities were highly comparable between the current and previous study for the nonfunctionalized MWCNTs. The generated adsorption isotherms and the fitted parameters contribute valuable information for comparison of adsorption behavior between the different CNT types in the M7 Elendt media.

The data showed a higher $Q^{0}$ of SWCNT compared to the MWCNTs. This is in accordance with our previous study using the same CNTs with algae medium and NOM ${ }^{11}$ and with other reports demonstrating a positive correlation between CNT SSA and $Q^{0.32}$ Interestingly, no relationship between CNT surface oxygen content and $Q^{0}$ was observed in the current study. This was further supported by the adsorption isotherms, which were similar for both functionalized and nonfunctionalized MWCNTs (Figure 1A). These data are in contrast to previous observations using the same CNTs in algae medium and $\mathrm{NOM}^{11}$ and to other studies where a decrease in $Q^{0}$ with increasing surface oxygen content has been observed. ${ }^{12,32}$ The $Q^{0}$ of the nonfunctionalized MWCNTs was very similar in both our current $(8.28 \mu \mathrm{g} / \mathrm{kg}$ and $8.37 \mu \mathrm{g} / \mathrm{kg}$ for MWCNT-15 and MWCNT-30, respectively) and previous studies $(8.22 \mu \mathrm{g} / \mathrm{kg}$ and $8.39 \mu \mathrm{g} / \mathrm{kg}$ for MWCNT-15 and MWCNT-30, respectively). However, a higher $Q^{0}$ was observed for the functionalized MWCNTs in the current study, with values being similar to those of the nonfunctionalized MWCNTs. For example, the $Q^{0}$ of MWCNT-COOH in the current study was $8.20 \mu \mathrm{g} / \mathrm{kg}$, compared to $7.64 \mu \mathrm{g} / \mathrm{kg}$ in the previous study. Normalizing the data against the estimated available surface area allows for further investigation of the effect of surface chemistry. Using data from our previous study, surface area normalized adsorption isotherms of the functionalized MWCNTs, in particular for MWCNT-COOH, differed from those of the nonfunctionalized MWCNTs (Figure S4). This indicated a suppression of phenanthrene adsorption due to the presence of oxygen containing surface functional groups. In the current study, normalizing the adsorption isotherms against the estimated available surface area resulted in very similar adsorption isotherms for all MWCNTs irrespective of surface chemistry (Figure 1B). In the M7-NOM medium, the presence of functional groups does not appear to influence phenanthrene adsorption to CNTs significantly. We have already demonstrated that media properties (ionic strength, concentration of divalent cations) can influence the role of CNT surface chemistry on CNT dispersibility. The current study also indicates that media properties are of importance with respect to the role of CNT surface chemistry on adsorption behavior. Media properties have been reported to affect adsorption of NOM to $\mathrm{CNTs},{ }^{33}$ but there is limited knowledge regarding the effect of media properties on the adsorption of HOCs, such as phenanthrene, to CNTs. Further studies are required to assess the role of aquatic media properties on adsorption behavior, as it would also have implications for the further effect of CNTs on cocontaminant bioavailability and the process of HOC adsorption in different aquatic environments.

In the current study, no clear relationship was observed between CNT properties and E. Similar observations were made in our previous study with the same CNTs in algae medium. ${ }^{11}$ It has been previously reported that $E$ of PAHs (pyrene) to CNTs varied with the type functionalization when dispersed by shaking. However, treatment with sonication removed differences in $E$ between different $C N T$ types but also resulted in an overall increase in $E$ for all CNT types. ${ }^{13}$ The presence of NOM has been shown to reduce $E .^{14}$ Despite the identical sonication treatment and SR-NOM concentration used in both our current and previous studies, ${ }^{11}$ slightly lower $E$ values were observed for all CNTs in the current study, in particular for the MWCNTs. Again, this suggests an influence of media properties on CNT adsorption behavior, which should receive attention in future studies.

Phenanthrene Toxicity to Daphnids. In all of the control exposures (pure Elendt M7, M7-NOM, and M7-NOM with methanol $<10 \%$ of the animals were immobilized, meeting with the OECD test criteria. This confirmed there was no 
toxicity that could be attributed to the M7 media, the presence of NOM, or from the methanol used for dissolving phenanthrene. There were no variations in $\mathrm{pH}$ outside the recommended range in any of the tests, and the $\mathrm{dO}_{2}$ concentration at the end of the experiment was well above the validation criteria $(>3 \mathrm{mg} / \mathrm{L})$ of the test guideline.

The percentage immobilization of daphnids as a function of phenanthrene concentration for the six individual tests performed is presented, together with the calculated $\mathrm{EC}_{50}$ values, in Figure S5. Although the $C_{\text {free }}$ (i.e., the measured phenanthrene concentration in the water phase) varied by $<10 \%$ from $C_{\text {nominal }}$ at all phenanthrene concentrations tested in the absence of CNTs, dose-response curves were calculated both for $C_{\text {nominal }}$ and $C_{\text {free. }}$ This was done as the response of $D$. magna to phenanthrene exposure was subsequently compared in the absence and presence of CNTs, both for $C_{\text {nominal }}$ and $C_{\text {free }}$. The $\mathrm{CV}$ of the calculated $\mathrm{EC}_{50}$ values for the six tests was $<6 \%$. As the variability between the tests was low, the data from the six tests were pooled in order to create an average doseresponse curve and determine $\mathrm{EC}_{50}$ values for phenanthrene. The average dose-response curves for phenanthrene-only are presented in Figure 2 (together with dose-response curves for
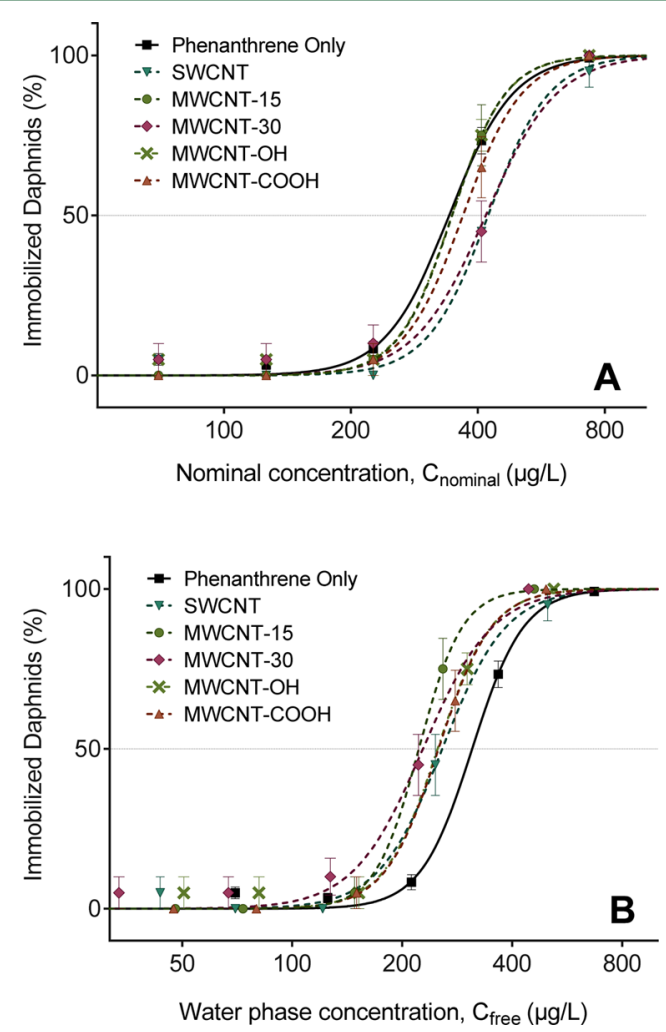

Figure 2. Immobilization of D. magna after exposure to phenanthrene in the absence and presence of CNTs. Phenanthrene concentrations are presented as $\mathrm{A}$ ) nominal concentrations $\left(C_{\text {nominal }}\right)$ and $\mathrm{B}$ ) freely dissolved concentrations $\left(C_{\text {free }}\right)$. Error bars represent the standard error of mean.

phenanthrene in the presence of CNTs), while the average $48 \mathrm{~h}$ $\mathrm{EC}_{50}$ value is shown in Table 2. Based on $C_{\text {nominal }}$ and $C_{\text {free }}$ the $\mathrm{EC}_{50}$ values were $342.1 \mu \mathrm{g} / \mathrm{L}\left(\mathrm{EC}_{50, \text { nominal }}\right)$ and $310.9 \mu \mathrm{g} / \mathrm{L}$ $\left(\mathrm{EC}_{50 \text {,free }}\right)$, respectively. The $48 \mathrm{~h} \mathrm{EC}_{50}$ for 3-4 day old daphnids exposed to phenanthrene was comparable to the $48 \mathrm{~h}$ $\mathrm{EC}_{50}$ values for $<24 \mathrm{~h}$ old daphnids previously reported (range from $230 \mu \mathrm{g} / \mathrm{L}$ to $550 \mu \mathrm{g} / \mathrm{L}) .^{25,35-37}$ Although we observed comparability with literature values for $<24 \mathrm{~h}$ old daphnids, a detailed assessment of the implications of using 3-4 day old daphnids was not tested in the current study.

Phenanthrene Toxicity in the Presence of CNTs. In control exposures containing CNTs-only (no phenanthrene), the immobilization of daphnids was $<10 \%$ in all cases, indicating there was no acute toxicity from any of the five CNT types at the concentrations tested (Table 1). Previous studies have reported a $50 \%$ mortality $\left(\mathrm{LC}_{50}\right)$ of $D$. magna $(<24$ h) exposed to MWCNTs dispersed in NOM at concentrations of $2-2.5 \mathrm{mg} / \mathrm{L} .^{18,38}$ These $\mathrm{LC}_{50}$ concentrations are comparable to the CNT concentrations used in the current study. However, they were achieved after a $96 \mathrm{~h}$ exposure time rather than the recommended $48 \mathrm{~h}$ exposure time in the test guideline. One of these studies also investigated D. magna $(<24 \mathrm{~h})$ mortality after $48 \mathrm{~h}$, observing no mortality even at $20 \mathrm{mg} / \mathrm{L}$ MWCNT exposure concentrations. ${ }^{38}$ The absence of an acute toxic response after $48 \mathrm{~h}$ and at the CNT concentrations employed in the current study therefore appears comparable to previous studies despite the difference in age of the D. magna used. The positive control samples contained daphnids exposed to phenanthrene-only (no CNTs) at concentrations representing the calculated $\mathrm{EC}_{30}$ and $\mathrm{EC}_{70}$ from the phenanthrene-only tests. These exhibited similar responses as those obtained in the phenanthrene-only tests, indicating no biological variability between the tests.

D. magna immobilization as a function of phenanthrene concentration in the presence of CNTs is presented in Figure 2, and the calculated $\mathrm{EC}_{50}$ values are presented in Table 2 . When using the $\mathrm{EC}_{50 \text {,nominal }}$ values, a significant reduction in phenanthrene toxicity $(p<0.003)$ was observed in the presence of SWCNT and MWCNT-30 compared to phenanthrene-only (Figure 2A, Table 2). For the three other CNTs (MWCNT-15, MWCNT-OH, and MWCNT-COOH), no significant difference in phenanthrene toxicity was observed in exposures with and without the presence of CNTs. Owing to the slightly higher dispersion concentration $(2.62 \mathrm{mg} / \mathrm{L})$ and available surface area $\left(0.46 \mathrm{~m}^{2} / \mathrm{L}\right)$ of MWCNT-30 compared to the other MWCNTs $\left(2.29-2.45 \mathrm{mg} / \mathrm{L}\right.$ and $0.32-0.34 \mathrm{~m}^{2} / \mathrm{L}$, respectively; Table 1), MWCNT-30 adsorbed a higher quantity of the added phenanthrene. As a result, $C_{\text {free }}$ was lower in the presence of MWCNT-30 than for the other MWCNTs (Figure S3). In the SWCNTs dispersions, the $C_{\text {free }}$ was also lower than the $C_{\text {free }}$ values determined for MWCNT-15, MWCNT-OH, and MWCNT-COOH for most of the $C_{\text {nominal }}$ phenanthrene values used in the study. As it was only the SWCNT and MWCNT-30 that reduced phenanthrene toxicity through adsorption, this could indicate that increased adsorption of phenanthrene by CNTs $\left(C_{\mathrm{CNT}}\right)$ causes a reduction in phenanthrene bioavailability to D. magna. Importantly, similar $\mathrm{EC}_{50}$ values were observed for both the phenanthrene-only exposures and the combined phenanthrene-CNT exposures for MWCNT-15, MWCNT-OH, and MWCNT-COOH despite a reduction in $C_{\text {free }}$ of $25-40 \%$ in these dispersions. These results indicate that phenanthrene adsorbed to CNTs remains bioavailable to $D$. magna. This bioavailability was further investigated by calculating dose-response curves for each CNT type based on their corresponding $C_{\text {free }}$ values (Figure 2B, Table 2). The data show a significant increase in phenanthrene toxicity in the presence of all CNT types when compared to phenanthrene-only. Therefore, the study strongly indicates that the phenanthrene adsorbed to all five different CNT types remained at least partially bioavailable and contributed directly 
Table 2. Calculated $\mathrm{EC}_{50}$ Values for Both $C_{\text {nominal }}\left(\mathrm{EC}_{50, \text { nominal }}\right)$ and $C_{\text {free }}\left(\mathrm{EC}_{50 \text {, free }}\right)$ Following Exposure to Phenanthrene-Only and Phenanthrene in the Presence of CNTs ${ }^{a}$

\begin{tabular}{|c|c|c|c|c|c|c|}
\hline $\mathrm{CNT}$ & $\mathrm{EC}_{50, \text { nominal }}(\mu \mathrm{g} / \mathrm{L})$ & $95 \% \mathrm{CI}$ & $R^{2}$ & $\mathrm{EC}_{50, \text { free }}(\mu \mathrm{g} / \mathrm{L})$ & $95 \% \mathrm{CI}$ & $R^{2}$ \\
\hline phenanthrene-only & $342.1^{\mathrm{a}}$ & $327.3-357.5$ & 0.9207 & $310.9^{\mathrm{a}}$ & $298.5-323.6$ & 0.9207 \\
\hline SWCNT & $423.2^{\mathrm{b}}$ & $393.1-455.7$ & 0.9373 & $257.5^{\mathrm{b}}$ & $235.6-281.4$ & 0.9373 \\
\hline MWCNT-15 & $347.8^{\mathrm{ab}}$ & $316.6-382.0$ & 0.9632 & $222.1^{\mathrm{b}}$ & $203.4-242.6$ & 0.9632 \\
\hline MWCNT-30 & $417.9^{\mathrm{b}}$ & $379.9-459.6$ & 0.9119 & $227.9^{\mathrm{b}}$ & $205.1-253.3$ & 0.9163 \\
\hline MWCNT-OH & $347.2^{\mathrm{ab}}$ & $316.4-381.0$ & 0.9602 & $250.0^{\mathrm{b}}$ & $224.4-278.5$ & 0.9601 \\
\hline MWCNT-COOH & $369.0^{\mathrm{ab}}$ & $340.8-399.5$ & 0.9601 & $251.3^{\mathrm{b}}$ & $231.1-273.3$ & 0.9599 \\
\hline
\end{tabular}

${ }^{a}$ Variations are presented as the $95 \%$ confidence interval $(\mathrm{CI})$ and the goodness of fit described by the coefficient of determination $\left(R^{2}\right)$. The $\mathrm{EC}_{50}$ values for phenanthrene-only are based on pooled data from six individual toxicity tests. Differing letters indicate statistical significant difference $(p<$ $0.05)$ within columns.

to the observed toxicity. Similarly, it has been observed that phenanthrene adsorbed to the fullerene $\mathrm{C}_{60}$ and to suspended sediment remain bioavailable and contribute to toxicity when exposed to D. magna. ${ }^{25,37}$ Moreover, phenanthrene absorbed to the same CNTs as used in the current study remained bioavailable to the algae $P$. subcapitata. ${ }^{11}$

No significant difference in toxicity was observed between any of the CNT types based on $C_{\text {free }}$ values. Thus, at similar $C_{\text {free }}$ values, the contribution to toxicity from CNT-adsorbed phenanthrene was comparable for all types of CNT. This indicates that CNT physicochemical properties did not significantly influence the mechanisms by which CNT-adsorbed phenanthrene was bioavailable or toxic to D. magna. It has been previously suggested that aggregation, expected to be higher for SWCNTs than MWCNTs due to the smaller diameter of SWCNTs, influences CNT elimination rates from the organisms and therefore affects $\mathrm{PAH}$ bioavailability to fish (Oryzias latipes and Pimephales promelas). ${ }^{19,24}$ Although the similar $\mathrm{EC}_{50 \text {,free }}$ values determined for each CNT type in the current study do not support this hypothesis, the importance of CNT aggregation behavior could vary for different organisms depending on their exposure and uptake routes. The data for $D$. magna are comparable to our previous study with freshwater algae ( $P$. subcapitata), where no clear relationship was observed between any of the CNT physicochemical properties evaluated and the $\mathrm{EC}_{50 \text {,free }}$ values determined. ${ }^{11}$ It is interesting to note that in both studies the hill slope of the dose-response curves were somewhat steeper for MWCNT-15 compared to the other CNTs and phenanthrene-only. ${ }^{11}$ This indicates there may be some variation in the bioavailability of phenanthrene in the presence of the different $\mathrm{CNTs}$, despite the similar $\mathrm{EC}_{50 \text {,free }}$ observed for all CNTs. However, this would need further investigation. The same observation regarding hill slope was made both for algae and for daphnids, suggesting this possible variation in phenanthrene bioavailability is independent of the organism exposed to the $\mathrm{CNT} /$ phenanthrene mixture and more likely related to one or more of the physicochemical differences between the test CNTs.

Optical microscopy images showed that CNTs were present in the digestive tracts of the daphnids (Figure 3). In some cases, CNT aggregates were observed attached to the surface of the daphnids; however, these were easily removed or dislodged by slight movement of the organisms with a pipet. As these surface-attached CNT aggregates were only occasionally observed, their influence on phenanthrene bioavailability and toxicity to D. magna is considered negligible when compared to the large quantity of ingested CNTs (observed in the gut). Uptake of PAHs through biological membranes and by pelagic invertebrates by passive diffusion has previously been

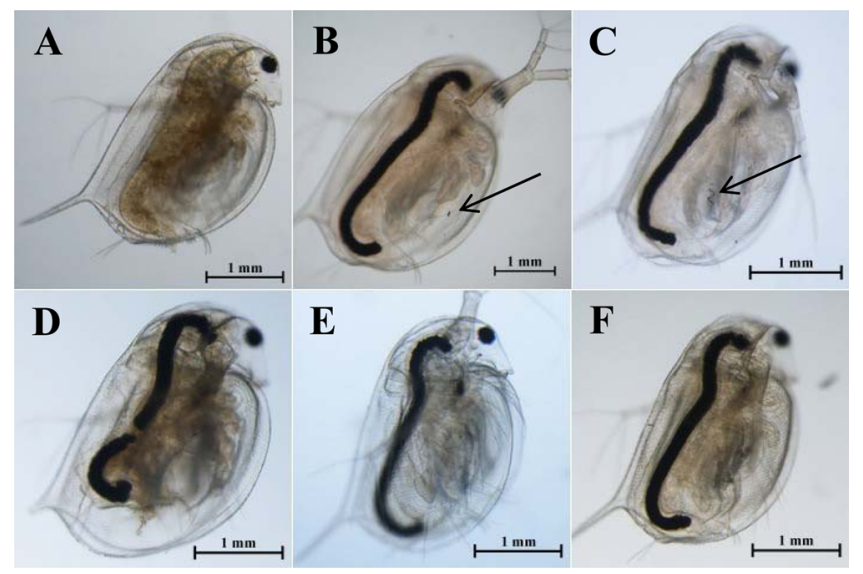

Figure 3. Light microscopy images $(\times 40)$ of $D$. magna after $48 \mathrm{~h}$ with A) no exposure or B-F) exposed to CNTs. (SWCNT, MWCNT-15, MWCNT-30, MWCNT-OH, and MWCNT-COOH for B, C, D, E, and $\mathrm{F}$, respectively.) The presence of CNTs in the digestive tract was clearly visible in exposed animals. Black arrows show CNT aggregates attached to the surface of the organisms.

reported. ${ }^{39,40}$ In the absence of CNTs, passive diffusion is considered the main uptake route of dissolved phenanthrene by D. magna in the current study. In the presence of CNTs, uptake by passive diffusion would be lower due to the lower $C_{\text {free }}$. Thus, data from the toxicity study indicate that another uptake route contributed to the toxicity. Ingestion of CNTs by daphnids appears to represent an alternative uptake route for phenanthrene, and the data suggest that CNT-adsorbed phenanthrene must be desorbing from the CNTs when passing through the digestive tract. Dissolved PAHs in aqueous systems will undergo adsorption or desorption to CNTs in order to establish equilibrium. ${ }^{41}$ When CNTs with adsorbed phenanthrene are ingested by D. magna, a lower (or most likely zero) phenanthrene concentration in the digestive tract will therefore promote desorption of phenanthrene from the CNTs into the dissolved phase where it is known to be bioavailable. Furthermore, the altered chemical environment in the digestive tract of D. magna might also promote desorption of phenanthrene from CNTs (e.g., $\mathrm{pH}$ which is 6.0-7.2 in daphnids ${ }^{42}$ ). The high $Q^{0}$ of CNTs implies that their presence could significantly alter the exposure routes of phenanthrene or other HOCs, increasing the likelihood of dietary exposure. An increased negative impact on end points such as reproduction and egg production in daphnids has been observed following dietary exposure and uptake to silver nanoparticles when compared to uptake of dissolved silver through passive diffusion from the water. ${ }^{43}$ However, the importance of dietary uptake 
with respect to uptake by passive diffusion for PAHs in $D$. magna is not known.

In the current study, no acute toxicity (immobilization) was observed due to the presence of the CNTs at any of the dispersion concentrations employed. However, sublethal effects caused by CNTs, and which may be compounded in the presence of phenanthrene, cannot be excluded. Furthermore, the presence of CNTs in the exposure system may facilitate an increased uptake or toxicological response to the dissolved phenanthrene fraction. Synergistic effects arising from the mixture of CNTs and phenanthrene could therefore represent another mechanism responsible for the increased toxicity based on $C_{\text {free }}$ observed in the current study. An insight into the role of mixture toxicity could be gained by investigating other, more sensitive, end points for CNT toxicity or by employing different CNT concentrations in a study where phenanthrene concentration remains constant. In the current study, no difference in toxicity was observed between the different types of CNTs when evaluating $C_{\text {free }}$. This implies that the contribution from the CNTs to any mixture toxicity effect should be similar for all CNT types, irrespective of their different properties and different dispersion concentrations (e.g., SWCNTs compared to MWCNTs). This would be in contrast to previous studies where a higher toxicity for nonfunctionalized MWCNTs compared to functionalized MWCNTs has been reported for daphnids (Ceriodaphnia dubia). ${ }^{44}$ Furthermore, comparable observations were made in our previous study where no difference in toxicity (based on $C_{\text {free }}$ ) was observed between CNT types. Although the combined effects of CNTs and phenanthrene could be a mechanism of toxicity, the data of our current and previous study suggest that this alone cannot explain the increased toxicity observed based on $C_{\text {free }}$.

There are several similarities between the toxicity data in our current study and our previous study with freshwater algae $(P$. subcapitata). ${ }^{11}$ Most importantly, in the previous study a significant reduction in algal toxicity was only observed when using $C_{\text {nominal }}$ values in the presence of SWCNT. However, when considering $C_{\text {free, }}$ an increase in algal toxicity compared to phenanthrene-only control samples was observed in the presence of all CNTs, similar to the observations made in the current study. ${ }^{11}$ These similarities were observed despite the fact that the exposure routes of CNT-adsorbed phenanthrene are significantly different for algae (e.g., dietary exposure is not relevant). Attachment of algal cells to CNT aggregates with adsorbed phenanthrene was believed to be of importance. In addition, a measurable reduction in $C_{\text {free }}$ due to the presence of algae was observed, implying that desorption of phenanthrene from CNTs could contribute to maintaining a higher $C_{\text {free }}{ }^{11}$ In contrast, the current study found there was no significant difference in $C_{\text {free }}$ in the presence and absence of $D$. magna in solutions with phenanthrene-only (Figure S6). Thus, desorption of phenanthrene from CNTs in the water phase is not believed to significantly influence $C_{\text {free }}$. It has been previously suggested that the presence of NOM impedes desorption of PAHs from CNTs, thus reducing the PAH bioavailability to fish. ${ }^{24}$ However, our current and previous studies ${ }^{11}$ suggest that the presence of NOM does not in general prevent bioavailability of PAHs adsorbed to CNTs, as the CNTadsorbed phenanthrene was bioavailable to both algae and daphnids despite the presence of NOM in these studies. However, the mechanisms of uptake and toxicity of CNTadsorbed PAHs are different in different species and trophic levels (e.g., algae, daphnids and fish). Both the mechanisms by which CNT-adsorbed phenanthrene contributes to toxicity for different organisms and the role of NOM in affecting the bioavailability of HOCs adsorbed by CNTs are of high environmental relevance and should be further investigated.

\section{ASSOCIATED CONTENT}

\section{S Supporting Information}

The Supporting Information is available free of charge on the ACS Publications website at DOI: 10.1021/acs.est.6b03228.

Tables showing physicochemical properties of the CNTs and fitted parameters of the Dubinin-Ashtakhov model and figures showing CNT calibration curves, $C_{\mathrm{CNT}}(\%)$ and $C_{\text {free }}$ as a function of $C_{\text {nominal }}$ adsorption isotherms from a previous study for comparison, dose-response curves from six tests addressing phenanthrene toxicity to D. magna, and measured phenanthrene concentration after $48 \mathrm{~h}$ exposure (PDF)

\section{AUTHOR INFORMATION}

\section{Corresponding Author}

*Phone: +47 93089510. E-mail: andy.booth@sintef.no.

\section{Present Address}

${ }^{\perp}$ Aquatic Ecology and Toxicology Section, Centre for Organismal Studies (COS), University of Heidelberg, Im Neuenheimer Feld 504, D-69120 Heidelberg, Germany.

\section{Notes}

The authors declare no competing financial interest.

\section{ACKNOWLEDGMENTS}

The work reported here has been undertaken as part of the Research Council of Norway (RCN) funded project "NanoSorb" (Grant Agreement number 209685/E50). The authors wish to thank the RCN for their financial support. We also wish to thank the External Cooperation Program of Chinese Academy of Sciences (Grant number GJHZ1206) for financial support. The authors acknowledge the essential technical assistance of Kristin Bonaunet, Lisbet Støen, Inger Steinsvik, Marianne Rønsberg, Kjersti Almås, Calin D. Marioara, John Walmsley, and Aud Spjelkavik (SINTEF Materials and Chemistry). We would also like to thank Gurvinder Singh for creating SEM images of the CNT materials.

\section{REFERENCES}

(1) De Volder, M. F. L.; Tawfick, S. H.; Baughman, R. H.; Hart, A. J. Carbon Nanotubes: Present and Future Commercial Applications. Science 2013, 339 (6119), 535-539.

(2) Yu, J. G.; Zhao, X. H.; Yang, H.; Chen, X. H.; Yang, Q.; Yu, L. Y.; Jiang, J. H.; Chen, X. Q. Aqueous adsorption and removal of organic contaminants by carbon nanotubes. Sci. Total Environ. 2014, 482-483, $241-251$.

(3) Koelmans, A. A.; Diepens, N. J.; Velzeboer, I.; Besseling, E.; Quik, J. T. K.; van de Meent, D. Guidance for the prognostic risk assessment of nanomaterials in aquatic ecosystems. Sci. Total Environ. 2015, 535, 141-149.

(4) Cerrillo, C.; Barandika, G.; Igartua, A.; Areitioaurtena, O.; Uranga, N.; Mendoza, G. Colloidal stability and ecotoxicity of multiwalled carbon nanotubes: Influence of select organic matters. Environ. Toxicol. Chem. 2016, 35 (1), 74-83.

(5) Hyung, H.; Fortner, J. D.; Hughes, J. B.; Kim, J. H. Natural organic matter stabilizes carbon nanotubes in the aqueous phase. Environ. Sci. Technol. 2007, 41 (1), 179-184.

(6) Schwyzer, I.; Kaegi, R.; Sigg, L.; Smajda, R.; Magrez, A.; Nowack, B. Long-term colloidal stability of 10 carbon nanotube types in the 
absence/presence of humic acid and calcium. Environ. Pollut. 2012, $169,64-73$.

(7) Peijnenburg, W. J. G. M.; Baalousha, M.; Chen, J.; Chaudry, Q.; Von der kammer, F.; Kuhlbusch, T. A. J.; Lead, J.; Nickel, C.; Quik, J. T. K.; Renker, M.; Wang, Z.; Koelmans, A. A. A Review of the Properties and Processes Determining the Fate of Engineered Nanomaterials in the Aquatic Environment. Crit. Rev. Environ. Sci. Technol. 2015, 45 (19), 2084-2134.

(8) Petersen, E. J.; Zhang, L. W.; Mattison, N. T.; O’Carroll, D. M.; Whelton, A. J.; Uddin, N.; Nguyen, T.; Huang, Q. G.; Henry, T. B.; Holbrook, R. D.; Chen, K. L. Potential Release Pathways, Environmental Fate, And Ecological Risks of Carbon Nanotubes. Environ. Sci. Technol. 2011, 45 (23), 9837-9856.

(9) Mauter, M. S.; Elimelech, M. Environmental applications of carbon-based nanomaterials. Environ. Sci. Technol. 2008, 42 (16), 5843-5859.

(10) Ren, X.; Chen, C.; Nagatsu, M.; Wang, X. Carbon nanotubes as adsorbents in environmental pollution management: A review. Chem. Eng. J. 2011, 170 (2-3), 395-410.

(11) Glomstad, B.; Altin, D.; Sørensen, L.; Liu, J.; Jenssen, B. M.; Booth, A. M. Carbon Nanotube Properties Influence Adsorption of Phenanthrene and Subsequent Bioavailability and Toxicity to Pseudokirchneriella subcapitata. Environ. Sci. Technol. 2016, 50 (5), 2660-2668.

(12) Wu, W. H.; Chen, W.; Lin, D. H.; Yang, K. Influence of Surface Oxidation of Multiwalled Carbon Nanotubes on the Adsorption Affinity and Capacity of Polar and Nonpolar Organic Compounds in Aqueous Phase. Environ. Sci. Technol. 2012, 46 (10), 5446-5454.

(13) Kah, M.; Zhang, X. R.; Hofmann, T. Sorption behavior of carbon nanotubes: Changes induced by functionalization, sonication and natural organic matter. Sci. Total Environ. 2014, 497-498, 133138.

(14) Zhang, X.; Kah, M.; Jonker, M. T. O.; Hofmann, T. Dispersion State and Humic Acids Concentration-Dependent Sorption of Pyrene to Carbon Nanotubes. Environ. Sci. Technol. 2012, 46 (13), 71667173.

(15) Pan, B.; Xing, B. Adsorption Mechanisms of Organic Chemicals on Carbon Nanotubes. Environ. Sci. Technol. 2008, 42 (24), 90059013.

(16) Petersen, E. J.; Akkanen, J.; Kukkonen, J. V. K.; Weber, W. J. Biological Uptake and Depuration of Carbon Nano-tubes by Daphnia magna. Environ. Sci. Technol. 2009, 43 (8), 2969-2975.

(17) Roberts, A. P.; Mount, A. S.; Seda, B.; Souther, J.; Qiao, R.; Lin, S. J.; Ke, P. C.; Rao, A. M.; Klaine, S. J. In vivo biomodification of lipidcoated carbon nanotubes by Daphnia magna. Environ. Sci. Technol. 2007, 41 (8), 3025-3029.

(18) Edgington, A. J.; Roberts, A. P.; Taylor, L. M.; Alloy, M. M.; Reppert, J.; Rao, A. M.; Mao, J. D.; Klaine, S. J. The Influence of natural organic matter on the toxicity of multiwalled carbon nanotubes. Environ. Toxicol. Chem. 2010, 29 (11), 2511-2518.

(19) Su, Y.; Yan, X. M.; Pu, Y. B.; Xiao, F.; Wang, D. S.; Yang, M. Risks of Single-Walled Carbon Nanotubes Acting as ContaminantsCarriers: Potential Release of Phenanthrene in Japanese Medaka (Oryzias latipes). Environ. Sci. Technol. 2013, 47 (9), 4704-4710.

(20) Alloy, M. M.; Roberts, A. P. Effects of suspended multi-walled carbon nanotubes on daphnid growth and reproduction. Ecotoxicol. Environ. Saf. 2011, 74 (7), 1839-1843.

(21) Long, Z. F.; Ji, J.; Yang, K.; Lin, D. H.; Wu, F. C. Systematic and Quantitative Investigation of the Mechanism of Carbon Nanotubes' Toxicity toward Algae. Environ. Sci. Technol. 2012, 46 (15), 84588466.

(22) Schwab, F.; Bucheli, T. D.; Lukhele, L. P.; Magrez, A.; Nowack, B.; Sigg, L.; Knauer, K. Are Carbon Nanotube Effects on Green Algae Caused by Shading and Agglomeration? Environ. Sci. Technol. 2011, 45 (14), 6136-6144.

(23) Smith, C. J.; Shaw, B. J.; Handy, R. D. Toxicity of single walled carbon nanotubes to rainbow trout, (Oncorhynchus mykiss): Respiratory toxicity, organ pathologies, and other physiological effects. Aquat. Toxicol. 2007, 82 (2), 94-109.
(24) Linard, E. N.; van den Hurk, P.; Karanfil, T.; Apul, O. G.; Klaine, S. J. Influence of carbon nanotubes on the bioavailability of fluoranthene. Environ. Toxicol. Chem. 2015, 34 (3), 658-666.

(25) Baun, A.; Sorensen, S. N.; Rasmussen, R. F.; Hartmann, N. B.; Koch, C. B. Toxicity and bioaccumulation of xenobiotic organic compounds in the presence of aqueous suspensions of aggregates of nano-C-60. Aquat. Toxicol. 2008, 86 (3), 379-387.

(26) Ferguson, P. L.; Chandler, G. T.; Templeton, R. C.; Demarco, A.; Scrivens, W. A.; Englehart, B. A. Influence of sediment-amendment with single-walled carbon nanotubes and diesel soot on bioaccumulation of hydrophobic organic contaminants by benthic invertebrates. Environ. Sci. Technol. 2008, 42 (10), 3879-3885.

(27) Schwab, F.; Bucheli, T. D.; Camenzuli, L.; Magrez, A.; Knauer, K.; Sigg, L.; Nowack, B. Diuron Sorbed to Carbon Nanotubes Exhibits Enhanced Toxicity to Chlorella vulgaris. Environ. Sci. Technol. 2013, 47 (13), 7012-7019.

(28) Shen, M.; Xia, X.; Zhai, Y.; Zhang, X.; Zhao, X.; Zhang, P. Influence of carbon nanotubes with preloaded and coexisting dissolved organic matter on the bioaccumulation of polycyclic aromatic hydrocarbons to Chironomus plumosus larvae in sediment. Environ. Toxicol. Chem. 2014, 33 (1), 182-189.

(29) OECD, Test No. 202: Daphnia sp. Acute Immobilisation Test. In OECD Guidelines for the Testing of Chemicals, Section 2; OECD Publishing: Paris, 2004.

(30) Yang, K.; Zhu, L. Z.; Xing, B. S. Adsorption of polycyclic aromatic hydrocarbons by carbon nanomaterials. Environ. Sci. Technol. 2006, 40 (6), 1855-1861.

(31) Glomstad, B.; Sørensen, L.; Liu, J.; Shen, M.; Zindler, F.; Jenssen, B. M.; booth, A. M. Evaluation of methods to determine adsorption of polycyclic aromatic hydrocarbons to dispersed carbon nanotubes 2016, Submitted.

(32) Yang, K.; Xing, B. Adsorption of Organic Compounds by Carbon Nanomaterials in Aqueous Phase: Polanyi Theory and Its Application. Chem. Rev. 2010, 110 (10), 5989-6008.

(33) Smith, B.; Yang, J.; Bitter, J. L.; Ball, W. P.; Fairbrother, D. H. Influence of Surface Oxygen on the Interactions of Carbon Nanotubes with Natural Organic Matter. Environ. Sci. Technol. 2012, 46 (23), 12839-12847.

(34) OECD, Test No. 201: Freshwater Alga and Cyanobacteria, Growth Inhibition Test. In OECD Guidelines for the Testing of Chemicals, Section 2, OECD Publishing: Paris, 2011.

(35) Smith, K. E. C.; Dom, N.; Blust, R.; Mayer, P. Controlling and maintaining exposure of hydrophobic organic compounds in aquatic toxicity tests by passive dosing. Aquat. Toxicol. 2010, 98 (1), 15-24.

(36) Wu, J.; Liu, Z.; Yan, Z.; Yi, X. Derivation of water quality criteria of phenanthrene using interspecies correlation estimation models for aquatic life in China. Environ. Sci. Pollut. Res. 2015, 22 (12), 94579463.

(37) Zhang, X.; Xia, X.; Dong, J.; Bao, Y.; Li, H. Enhancement of toxic effects of phenanthrene to Daphnia magna due to the presence of suspended sediment. Chemosphere 2014, 104, 162-169.

(38) Kim, K.-T.; Edgington, A. J.; Klaine, S. J.; Cho, J.-W.; Kim, S. D. Influence of Multiwalled Carbon Nanotubes Dispersed in Natural Organic Matter on Speciation and Bioavailability of Copper. Environ. Sci. Technol. 2009, 43 (23), 8979-8984.

(39) Bugg, T.; Foght, J. M.; Pickard, M. A.; Gray, M. R. Uptake and active efflux of polycyclic aromatic hydrocarbons by Pseudomonas fluorescens LP6a. Appl. Environ. Microbiol. 2000, 66 (12), 5387-5392.

(40) Meador, J. P.; Stein, J. E.; Reichert, W. L.; Varanasi, U. Bioaccumulation of Polycyclic Aromatic Hydrocarbons by Marine Organisms. Rev. Environ. Contam. Toxicol. 1995, 143, 79-165.

(41) Yang, K.; Xing, B. S. Desorption of polycyclic aromatic hydrocarbons from carbon nanomaterials in water. Environ. Pollut. 2007, 145 (2), 529-537.

(42) Ebert, D. Introduction to Daphnia Biology. In Ecology, Epidemiology, and Evolution of Parasitism in Daphnia; National Center for Biotechnology Information: Bethesda, MD, USA, 2005. 
(43) Hook, S. E.; Fisher, N. S. Sublethal effects of silver in zooplankton: Importance of exposure pathways and implications for toxicity testing. Environ. Toxicol. Chem. 2001, 20 (3), 568-574.

(44) Kennedy, A. J.; Hull, M. S.; Steevens, J. A.; Dontsova, K. M.; Chappell, M. A.; Gunter, J. C.; Weiss, C. A. Factors influencing the partitioning and toxicity of nanotubes in the aquatic environment. Environ. Toxicol. Chem. 2008, 27 (9), 1932-1941. 